\title{
Hyperactivated mTORC1 downregulation of FOX03a/PDGFRa/ AKT cascade restrains tuberous sclerosis complex-associated tumor development
}

\author{
Li Wang ${ }^{1, *}$, Zhaofei $\mathrm{Ni}^{1, *}{ }^{*}$, Yujie Liu' ${ }^{2,}{ }^{*}$, Shuang $\mathrm{Ji}^{1}$, Fuquan Jin ${ }^{1}$, Keguo Jiang ${ }^{3}$, Junfang $\mathrm{Ma}^{4}$, \\ Cuiping Ren ${ }^{5}$, Hongbing Zhang ${ }^{6}$, Zhongdong $\mathrm{Hu}^{7}$ and Xiaojun $\mathrm{Zha}^{1}$ \\ ${ }^{1}$ Department of Biochemistry and Molecular Biology, School of Basic Medicine, Anhui Medical University, Hefei, China \\ ${ }^{2}$ The First Clinical Medical School, Anhui Medical University, Hefei, China \\ ${ }^{3}$ Department of Nephrology, The Third Affiliated Hospital of Anhui Medical University, Hefei, China \\ ${ }^{4}$ Department of Neurology, Beijing Shijitan Hospital, Capital Medical University, Beijing, China \\ ${ }^{5}$ Department of Parasitology, School of Basic Medicine, Anhui Medical University, Hefei, China \\ ${ }^{6}$ State Key Laboratory of Medical Molecular Biology, Department of Physiology and Pathophysiology, Institute of Basic Medical \\ Sciences, Chinese Academy of Medical Sciences and Peking Union Medical College, Beijing, China \\ ${ }^{7}$ Modern Research Center for Traditional Chinese Medicine, School of Chinese Materia Medica, Beijing University of Chinese \\ Medicine, Beijing, China \\ *These authors contributed equally to this work
}

Correspondence to: Xiaojun Zha, email: zhaxiaojunpumc@gmail.com Zhongdong Hu, email: zdhu@bucm.edu.cn

Keywords: mTOR, FOXO3a, PDGFRa, AKT, tumorigenesis

Received: January 30, 2017 Accepted: June 18, 2017

Published: July 04, 2017

Copyright: Wang et al. This is an open-access article distributed under the terms of the Creative Commons Attribution License 3.0 (CC BY 3.0 ), which permits unrestricted use, distribution, and reproduction in any medium, provided the original author and source are credited.

\section{ABSTRACT}

Hyperactivation of mammalian target of rapamycin complex 1 (mTORC1), caused by loss-of-function mutations in either the TSC1 or TSC2 gene, leads to the development of tuberous sclerosis complex (TSC), a benign tumor syndrome with multiple affected organs. mTORC1-mediated inhibition of AKT constrains the tumor progression of TSC, but the exact mechanisms remain unclear. Herein we showed that loss of TSC1 or TSC2 downregulation of platelet-derived growth factor receptor a (PDGFRa) expression was mediated by mTORC1. Moreover, mTORC1 inhibited PDGFRa expression via suppression of forkhead box 03a (FOX03a)-mediated PDGFRa gene transcription. In addition, ectopic expression of PDGFRa promoted AKT activation and enhanced proliferation and tumorigenic capacity of Tsc1- or Tsc2-null mouse embryonic fibroblasts (MEFs), and vice versa. Most importantly, rapamycin in combination with AG1295, a PDGFR inhibitor, significantly inhibited growth of TSC1/ TSC2 complex-deficient cells in vitro and in vivo. Therefore, downregulated FOXO3a/ PDGFRa/AKT pathway exerts a protective effect against hyperactivated mTORC1induced tumorigenesis caused by loss of TSC1/TSC2 complex, and the combination of rapamycin and AG1295 may be a new effective strategy for TSC-associated tumors treatment.

\section{INTRODUCTION}

The mammalian target of rapamycin (mTOR) is a conserved serine/threonine protein kinase implicated in a wide array of cellular processes including cell proliferation, growth, metabolism, and autophagy [1-4].
mTOR forms at least two distinct multi-protein complexes, mTOR complex 1 (mTORC1) and mTOR complex 2 (mTORC2). mTORC1 is composed of mTOR, Raptor, mLST8, PRAS40, and Deptor, which is inhibited by rapamycin and its analogs, whereas mTORC2 consists of $\mathrm{mTOR}$, Rictor, and several other binding proteins and is 
rapamycin-insensitive [5-7]. mTORC1 integrates a wide range of upstream signals from growth factors, nutrients, oxygen, and energy status to orchestrate cell growth, metabolism, and survival $[1,8]$.

Tuberous sclerosis complex (TSC) is an autosomal dominant disorder characterized by the formation of benign tumors in multiple organs including kidney, brain, skin, and heart, which is caused by inactivating mutations in either of two tumor suppressor genes: TSC1 or TSC2 [9-11]. TSC1 and TSC2 form a functional complex that is a suppressor upstream of mTORC1 $[12,13]$. Lossof-function mutations in either the TSC1 or TSC2 gene lead to activation of mTORC1, which is believed to be responsible for the tumor development in TSC [9, 14-16]. Interestingly, patients with TSC rarely develop malignant lesions. It has been reported that mTORC1-mediated negative feedback inhibition of AKT contributes to the benign nature of TSC tumors [17-20]. However, the underlying mechanisms remain poorly understood.

Platelet-derived growth factor (PDGF) stimulates cell proliferation, survival, angiogenesis, and migration via binding to $\alpha$ and $\beta$ tyrosine kinase receptors, PDGFR $\alpha$ and PDGFR $\beta[21,22]$. It has been reported that PDGF-related signaling is linked to the pathogenesis of fibrotic disorders, atherosclerosis, and cancers [18, 23]. Intriguingly, PDGFR $\alpha$ and PDGFR $\beta$ show distinct effects in the development of human diseases [24-26]. We have shown that loss of TSC1/TSC2 complex reduced the expression of PDGFR $\alpha$ and PDGFR $\beta$ [17], and restoration of PDGFR $\beta$ enhanced tumorigenic potential of TSC1/ TSC2 complex-deficient cells [18]. However, the function and regulation of PDGFR $\alpha$ in TSC-associated tumors remain elusive.

In this study, we demonstrated that hyperactivated mTORC1 downregulated PDGFR $\alpha$ expression through inhibition of forkhead box O3a (FOXO3a), which led to inactivation of $\mathrm{AKT}$ and subsequently attenuated the tumorigenicity of Tsc1- or Tsc2-null mouse embryonic fibroblasts (MEFs). In addition, the combination of rapamycin and AG1295, a specific PDGFR inhibitor, may be exploited as a novel regimen for the treatment of TSCrelated cancers.

\section{RESULTS}

\section{Loss of TSC1 or TSC2 reduced PDGFRa expression through activation of $\mathrm{mTORC1}$}

Consistent with previous studies [17, 18], loss of TSC1 or TSC2 induced mTORC1 activation (p-S6 is an indicator of mTORC1 activity) and AKT inhibition (Figure 1A and 1B). Also, loss of TSC1 or TSC2 led to decreased expression of $\operatorname{PDGFR} \alpha$ at both protein and mRNA levels (Figure 1A and 1B). To investigate whether mTORC1 is involved in the regulation of PDGFR $\alpha$ expression, we first examined the effect of rapamycin, a specific mTORC1 inhibitor, on PDGFR $\alpha$ expression. As shown in Figure $1 \mathrm{C}$ and $1 \mathrm{D}$, rapamycin treatment markedly increased PDGFR $\alpha$ expression in Tsc2-/or Tsc1-/- MEFs. To further verify that the negative regulation of PDGFR $\alpha$ by loss of TSC1/TSC 2 complex is mediated by mTORC1, we examined PDGFR $\alpha$ levels in Tsc2-/- or Tsc1-/- MEFs with knockdown of Raptor (a specific component of mTORC1). As shown in Figure $1 \mathrm{E}$ and $1 \mathrm{~F}$, depletion of Raptor with siRNAs increased PDGFR $\alpha$ levels in Tsc2- or Tsc1-null MEFs. However, knockdown of Rictor (a specific component of mTORC2) had a minimal effect on the expression of PDGFR $\alpha$ in Tsc2- or Tsc1-null MEFs (Supplementary Figure 1). Taken together, hyperactivated $\mathrm{mTORC} 1$ is responsible for the downregulation of PDGFR $\alpha$ due to loss of TSC1/TSC2 complex.

\section{Decreased PDGFR $\alpha$ blunts the proliferation and tumorigenesis of Tsc1- or Tsc2-deficient cells}

To evaluate the role of PDGFR $\alpha$ in TSC tumors, Tsc2-null MEFs were infected with lentiviruses for overexpressing PDGFR $\alpha$, which was confirmed by western blot analysis (Figure 2A). The ectopic PDGFR $\alpha$ expression markedly enhanced cell proliferation (Figure 2D up panel) and colony formation (Figure 2E up panel). Consistent results were obtained in Tsc1-deficient MEFs overexpressing PDGFR $\alpha$ (Figure 2B, 2D middle panel, and $2 \mathrm{E}$ middle panel). Moreover, depletion of PDGFR $\alpha$ using shRNAs targeting PDGFR $\alpha(\operatorname{shPDGFR} \alpha)$ in Tsc $2+/+$ MEFs inhibited cell proliferation and colony formation (Figure 2C, 2D low panel, and 2E low panel).

Next we investigated the role of PDGFR $\alpha$ in TSC tumors in vivo. Tsc2-/- MEFs transfected with lentiviral vector encoding PDGFR $\alpha$ or empty vector were subcutaneously injected into the right anterior armpit of nude mice, and then tumor growth was monitored. As depicted in Figure 2F, overexpression of PDGFR $\alpha$ dramatically enhanced the tumorigenic capacity of Tsc2-/- MEFs. IHC analysis revealed that tumor tissues derived from mice with injection of PDGFR $\alpha$ overexpressing Tsc2-/- MEFs exhibited much stronger staining for $\mathrm{Ki}-67$ than those in the control group (Figure 2H). Consistent results were obtained in mice with injection of PDGFR $\alpha$-overexpressing Tsc1-/- MEFs and control mice (Figure $2 \mathrm{G}$ and $2 \mathrm{I}$ ).

\section{PDGFRa is essential for AKT activation}

Since the activation of mTORC1 led to downregulated expression of PDGFR $\alpha$ and inhibition of AKT in Tsc1- or Tsc2-null MEFs, thus we speculated that decreased PDGFR $\alpha$ is, at least in part, responsible for the attenuated AKT activity due to hyperactivation of mTORC1. To test this hypothesis, Tsc2-/- MEFs with overexpression of PDGFR $\alpha$ and the control cells were 
starved and then treated with fetal bovine serum (FBS) or PDGF for up to $30 \mathrm{~min}$. As shown in Figure 3A, both serum and PDGF stimulation enhanced the activation of AKT induced by PDGFR $\alpha$ overexpression in Tsc2-/MEFs. Similarly, PDGFR $\alpha$ overexpression also augmented serum or PDGF-stimulated AKT activation in Tsc1-/MEFs (Figure 3B). Moreover, reduction of PDGFR $\alpha$ markedly attenuated AKT activation induced by serum or PDGF in Tsc2+/+ or Tsc1+/+ MEFs (Figure 3C and 3D). Furthermore, an elevated level of p-AKT was observed in tumor tissues derived from PDGFR $\alpha$-overexpressing Tsc2-/- or Tsc1-/- MEFs as compared to the control tissues (Figure 3E and 3F). Collectively, these data reveal that PDGFR $\alpha$ is critical for AKT activation and loss of TSC1/TSC2 complex downregulates AKT activity partially through inhibition of PDGFR $\alpha$ expression.

\section{mTORC1 negatively regulates FOXO3a in Tsc1- or Tsc2-null MEFs}

The transcription factor FOXO3a is a positive regulator of cell apoptosis and cell cycle arrest and is frequently dysregulated in cancer $[32,33]$. In many cancer cells, FOXO3a is phosphorylated and translocated to the
A

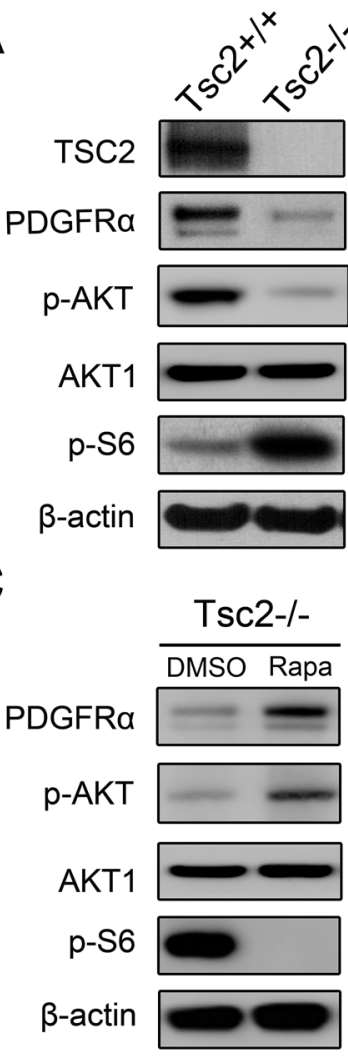

$E$

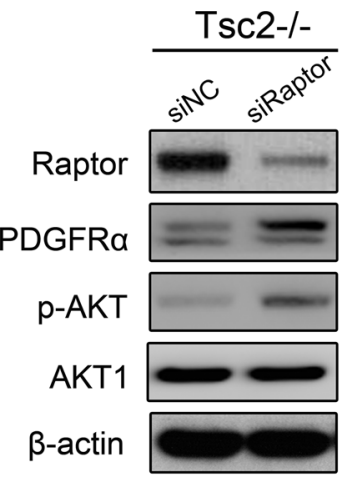


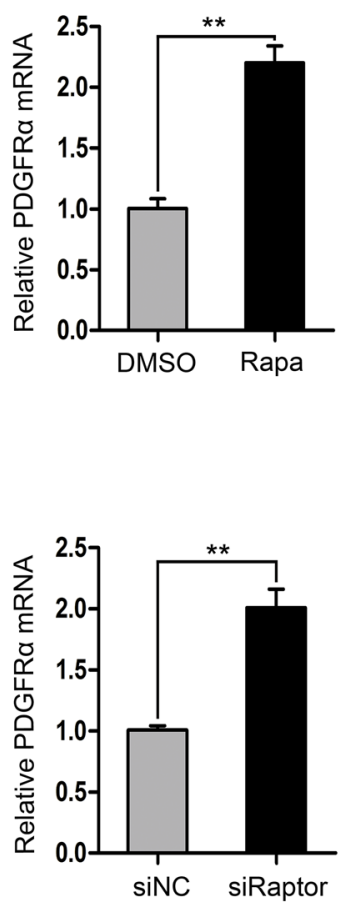

B


$\mathrm{D}$

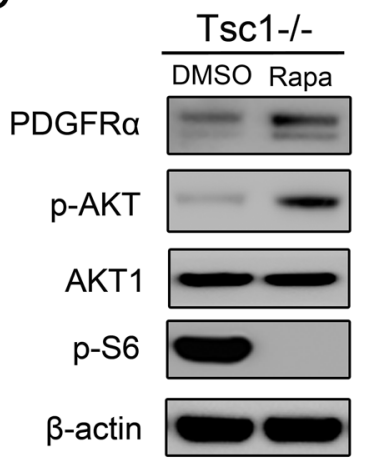

$\mathrm{F}$
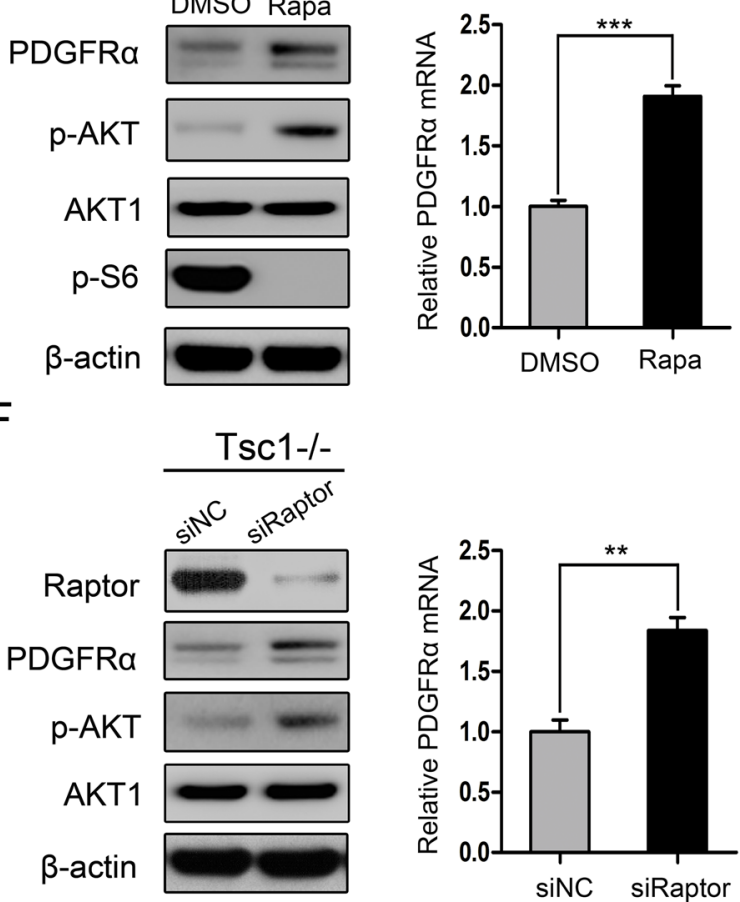

Figure 1: mTORC1 is a negative regulator of PDGFRa. (A) Tsc2+/+ and Tsc2-/- MEFs. (B) Tsc1+/+ and Tsc1-/- MEFs. (C, D) Tsc2-/- (C) or Tsc1-/- MEFs (D) were treated with DMSO or with $20 \mathrm{nM}$ rapamycin (Rapa) for $24 \mathrm{~h}$. (E, F) Tsc2-/-(E) or Tsc1-/- MEFs (F) were transfected with the control siRNAs (siNC) or siRNAs targeting Raptor for $48 \mathrm{~h}$. A-F. Cell lysates were subjected to immunoblotting with the indicated antibodies (left panels). qRT-PCR was performed to detect the mRNA level of PDGFRa (right panels). ${ }^{* * P} P<0.01 ; * * * P<0.001$. 
A

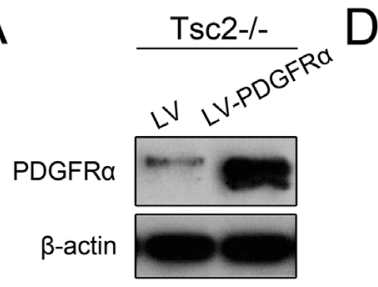

B

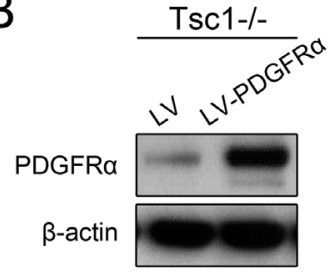

C

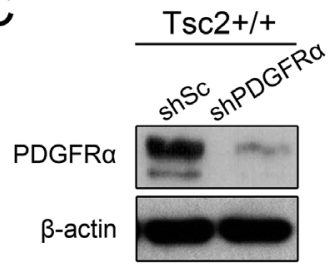

E
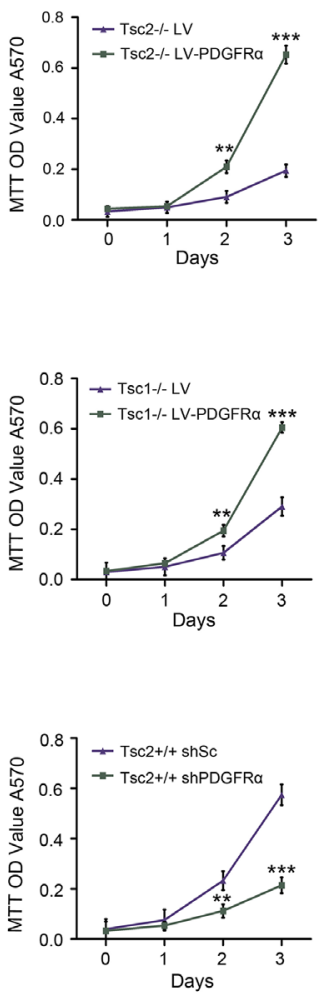

F

Tsc2-I-

LV

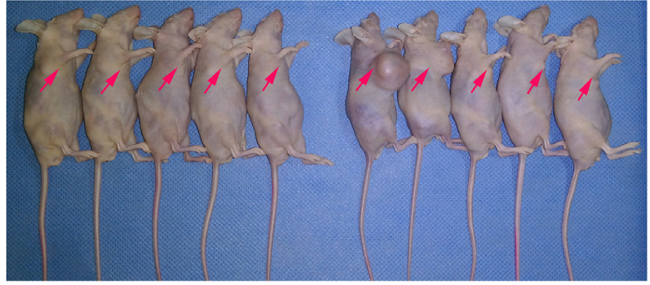

$\mathrm{H}$

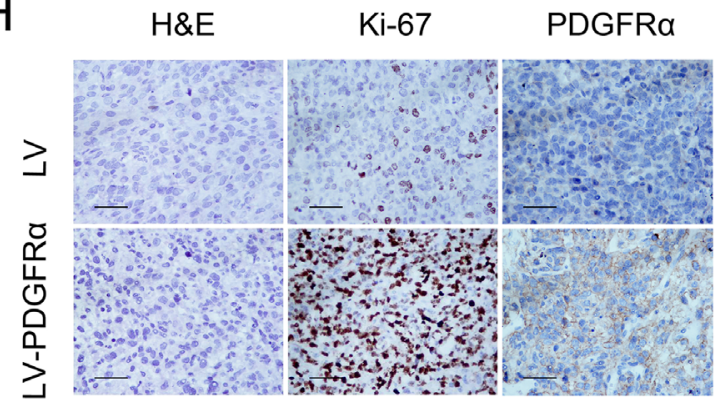

G

1
3
3
0
0
$\frac{1}{4}$
0
0
0
3
3
LV LV-PDGFR $\alpha$



Tsc2-/-

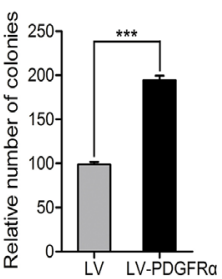

LV LV-PDGFRa



Tsc1-/-


Tsc2+/+

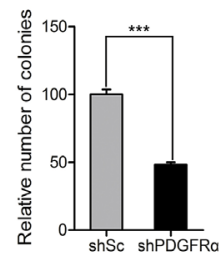

Tsc1-/-

LV

LV-PDGFRa

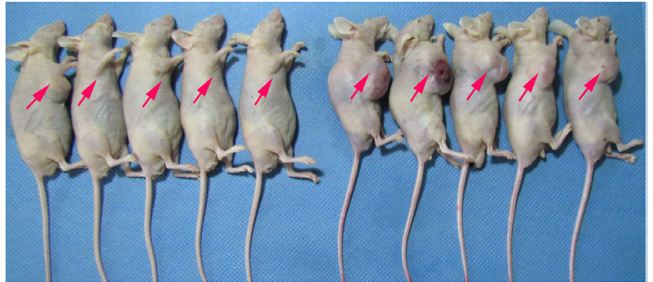

H\&E

Ki-67

PDGFR $\alpha$

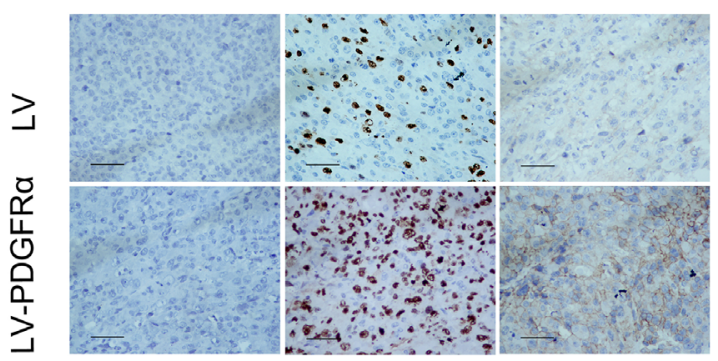

Figure 2: Decreased PDGFRa inhibits the growth of cells with deficiency of TSC1/TSC2 complex in vitro and in vivo. $(\mathbf{A}, \mathbf{B})$ Tsc2-/- (A) or Tsc1-/- MEFs (B) were infected with lentivirus harboring a vector encoding PDGFRa (LV-PDGFRa) or the empty vector (LV). (C) Tsc2+/+ MEFs stably expressing shRNAs targeting PDGFRa (shPDGFRa) or a control shRNA (shSc). A-C. Cell lysates were subjected to immunoblotting with the indicated antibodies. (D) Proliferation of the indicated cells was examined using an MTT assay. (E) The colonies formed by the indicated cells were stained and counted. Representative images (left panels) and quantifications (right panels). (F, G) Tsc2-l-(F) or Tsc1-/- MEFs (G) transduced with LV-PDGFRa or LV lentiviruses were inoculated subcutaneously into nude mice, followed by monitoring for tumor growth. (H, I) Tumor tissues derived from Tsc2-/- (H) or Tsc1-/- MEFs (I) were fixed and embedded with paraffin, and then subjected to $H \& E$ and immunohistochemical staining. Representative images were presented. Scale bar, $50 \mu \mathrm{m} . * * P<0.01 ; * * * P<0.001$. 
cytoplasm due to aberrantly activated AKT, leading to inhibition of target gene transcription [32, 34]. As shown in Figure 4A, both p-FOXO3a (Ser253) and total FOXO3a were dramatically decreased in cells lacking TSC1/TSC2 complex as comparison with the control cells and were partially rescued by mTORC1 inhibition. Interestingly, nucleocytoplasmic separation experiments (Figure 4B) and immunofluorescence analysis (Figure 4C) showed that the nuclear level of FOXO3a was also dramatically reduced in Tsc2-/- MEFs and was markedly elevated by

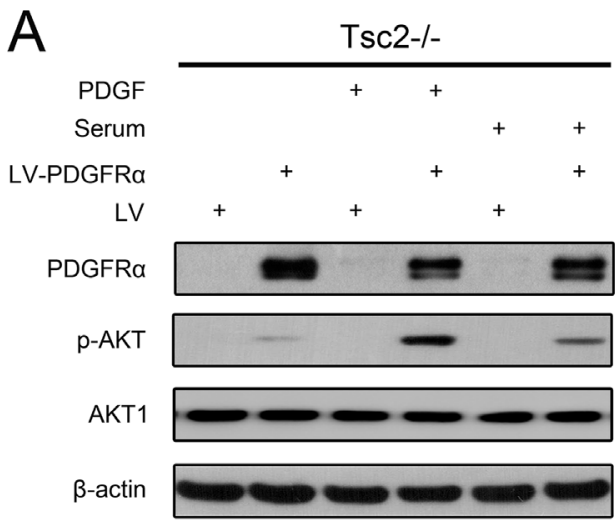

C


rapamycin treatment. These findings indicate that both the expression level and the nuclear accumulation of FOXO3a are negatively regulated by $\mathrm{mTORC1}$, regardless of AKT activity. Furthermore, luciferase reporter assay confirmed that the transcriptional and DNA-binding activity of FOXO3a was significantly decreased in Tsc2null MEFs and was rescued by rapamycin (Figure 4D). A consistent result was obtained in Tsc1-/- MEFs in response to rapamycin treatment (Figure 4E). In addition, we also determined whether FOXO3a is controlled by
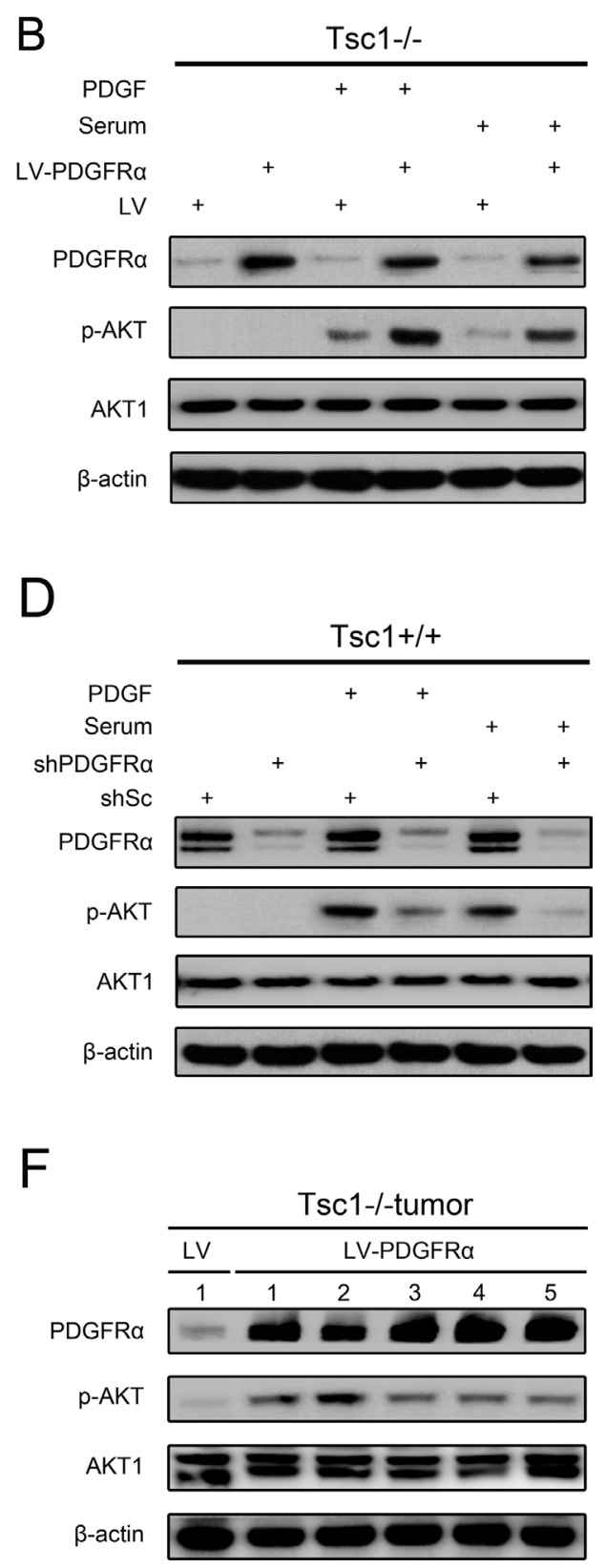

Figure 3: PDGFRa is essential for AKT activation. (A, B) Tsc2-/- (A) or Tsc1-/- MEFs (B) were transduced with LV-PDGFRa or LV lentiviruses. (C, D) Tsc2+/+ (C) or Tsc1+/+ MEFs (D) were transduced with shPDGFRa or shSc lentiviruses. A-D. Cells were starved in DMEM for $24 \mathrm{~h}$, followed by stimulation with serum or PDGF $(50 \mathrm{ng} / \mathrm{ml})$ for $30 \mathrm{~min}$, and then cell lysates were harvested and subjected to immunoblotting with the indicated antibodies. (E, F) Tumor tissues derived from Tsc2-/- (E) or Tsc1-/- MEFs (F) transduced with LVPDGFRa or LV lentiviruses were subjected to immunoblotting with the indicated antibodies. 
mTORC1 by using lentiviral vectors expressing shRNAs targeting Raptor in Tsc2-/- MEFs. As shown in Figure 4F, knockdown of Raptor strikingly decreased mTORC1 activity and increased the expression of $\mathrm{p}$-AKT. Depletion of Raptor led to dramatically upregulated both total FOXO3a and nuclear FOXO3a, and significantly increased FOXO3a transcriptional activity (Figure 4F, 4G and 4H). Similarly, reduction of Raptor dramatically increased nuclear FOXO3a and its transcriptional activity in Tsc1-/MEFs (Figure 4I). Taken together, both expression level and activity of $\mathrm{FOXO} 3 \mathrm{a}$ were negatively regulated by mTORC1 in cells lacking TSC1/TSC2 complex.

\section{mTORC1 downregulates PDGFRa/AKT pathway through suppression of FOXO3a}

Activation of mTORC1 led to both inhibition of FOXO3a and downregulated PDGFR $\alpha$ expression, so we next investigated whether mTORC1 downregulation of PDGFR $\alpha$ is mediated by FOXO3a. Tsc2- or Tsc1-null MEFs were transfected with lentiviruses overexpressing FOXO3aTM (a constitutively active FOXO3a). Ectopic expression of FOXO3aTM significantly promoted the expression of PDGFR $\alpha$ and p-AKT in Tsc2-/- or Tsc1-/MEFs (Figure 5A upper panels). qRT-PCR analysis showed that FOXO3a upregulated the mRNA level of PDGFR $\alpha$ (Figure 5A lower panels). Conversely, depletion of FOXO3a with RNA interference decreased the levels of PDGFR $\alpha$ and p-AKT in Tsc2+/+ or Tsc1+/+ MEFs (Figure 5B). Collectively, mTORC1 downregulates PDGFR $\alpha / A K T$ signaling cascade via inhibition of FOXO3a.

To further elucidate the mechanisms underlying FOXO3a regulation of PDGFR $\alpha$, we analyzed the 5 '-flanking sequence of the PDGFR $\alpha$ gene upstream of the transcription start site. Two conserved FOXO3a binding sequences (-441/-435 GCAAACA; -264/-258 GAAAACA) were identified within the promoter of the mouse PDGFR $\alpha$ gene (Figure 5C). We then cloned the mouse PDGFR $\alpha$ gene promoter $(-582 /+68)$ into a luciferase reporter vector and evaluated the effect of FOXO3a on the promoter activity. Luciferase reporter assay in HEK293T cells showed that overexpression of FOXO3aTM increased luciferase activity (Figure 5D), indicating that $\mathrm{FOXO} 3 \mathrm{a}$ directly regulates $\mathrm{PDGFR} \alpha$ transcription. Moreover, the enhanced transcriptional activity was attenuated when either of the two putative FOXO3a-binding sequences were mutated (Figure 5E). ChIP analysis further revealed that FOXO3a bound to these two putative sites (Figure 5F). In addition, the direct binding of FOXO3a to the two putative sites within the PDGFR $\alpha$ gene promoter was drastically weaker in Tsc1-/- MEFs than in the control cells, and was rescued by suppression of mTORC1 with rapamycin (Figure 5G). Taken together, FOXO3a promotes the transcription of PDGFR $\alpha$ through directly binding to the promoter of PDGFR $\alpha$ gene.

\section{Rapamycin in combination with AG1295 inhibits the growth of cells lacking TSC1/TSC2 complex in vitro and in vivo}

Since the inhibition of mTORC1 markedly increased PDGFR $\alpha$ expression and then led to activation of AKT, we next evaluated whether the combination of rapamycin with AG1295 (a specific PDGFR inhibitor) could achieve a better inhibitory effect on the growth of cells lacking TSC1/TSC2 complex. As depicted in Figure 6A, the combination treatment of rapamycin and AG1295 exerted a stronger inhibitory effect on cell viability of Tsc2-/- MEFs than either compound alone, which was consistent with the result in Tsc1-/- MEFs (Figure 6B). Next we explored the in vivo effect of combination administration of rapamycin and AG1295 by using a mouse tumor model with NTC/ T2-null cells (a cell line with potent tumorigenicity derived from Tsc2-/- MEFs) [28]. As shown in Figure 6C-6E, the tumor volumes and tumor weights were significantly inhibited by treatment with rapamycin or AG1295 alone, and rapamycin in combination with AG1295 exerted a better anti-tumor activity than the treatment with either drug alone. Moreover, the combination treatment did not significantly affect the body weights of the mice (Figure 6F). Therefore, AG1295 enhances the inhibitory effect of rapamycin on the tumorigenic capacity of cells lacking TSC1/TSC2 complex.

\section{DISCUSSION}

Overactivation of AKT is a hallmark of many human tumors [35]. Although TSC is a genetic disease with tumors formation in multiple organs [9], AKT activity is reduced in TSC tumor cells [17-20]. This phenomenon contributes to explain why most TSC patients are inclined to develop benign tumors [9, 36-38]. However, the precise mechanisms underlying the reduced AKT activity due to loss of TSC1/TSC2 complex remain incompletely understood. It has been suggested that mTORC1-mediated regulation of several molecules, such as Grb10, IRS, and PTEN, are involved in the suppression of AKT activity in TSC cells [18, 20, 39]. Recently, we have also identified an mTORC1-independent manner which mediates AKT inhibition in TSC lesions [40]. Here we showed that loss of TSC1/TSC2 complex downregulated PDGFR $\alpha$ expression via activation of mTORC1 (Figure 1). Ectopic expression of PDGFR $\alpha$ enhanced serum- or PDGFstimulated AKT activation in Tsc1- or Tsc2-null MEF cells (Figure 3). Moreover, overexpression of PDGFR $\alpha$ accelerated cell proliferation and tumor growth of Tsc1or Tsc2-null MEFs (Figure 2). Thus, mTORC1 suppresses AKT activity through downregulation of PDGFR $\alpha$, which ameliorates the malignancy of TSC.

Although mTORC1 is recognized as the pivotal regulator of protein translation, accumulating evidence shows that mTORC1 affects gene expression through 
A

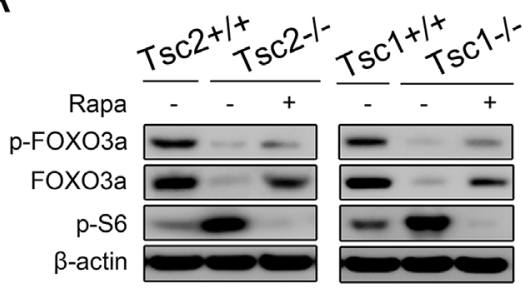

B

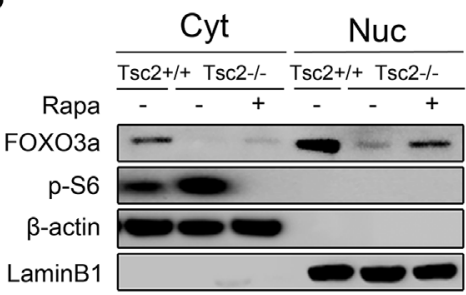

D

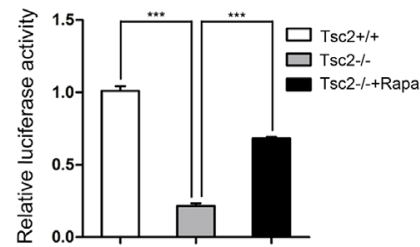

F

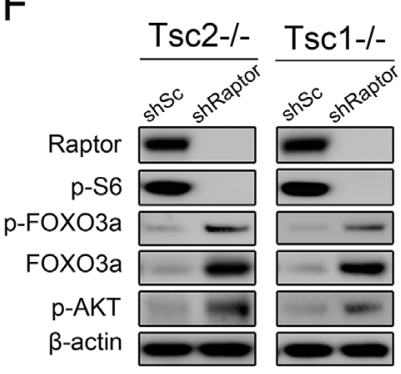

$\mathrm{H}$

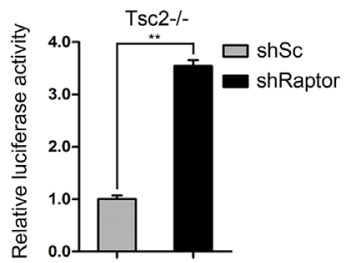

C

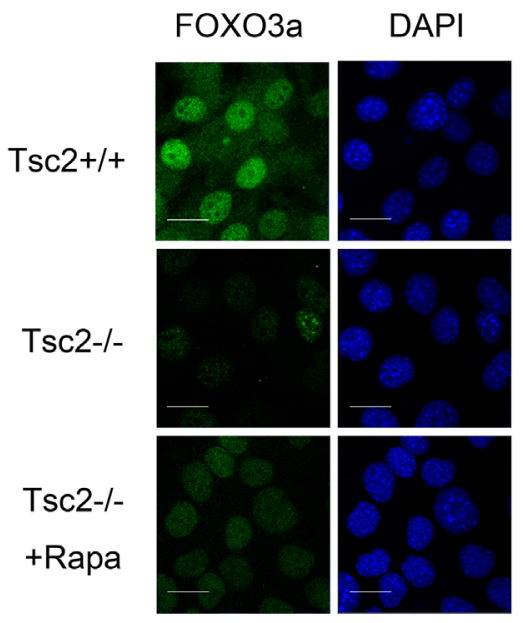

E

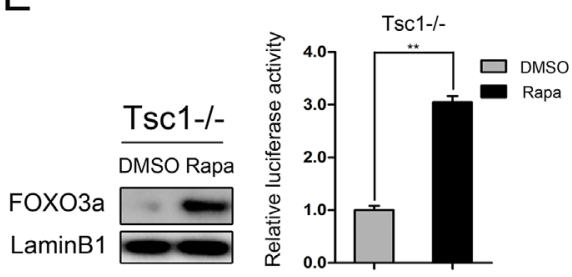

G

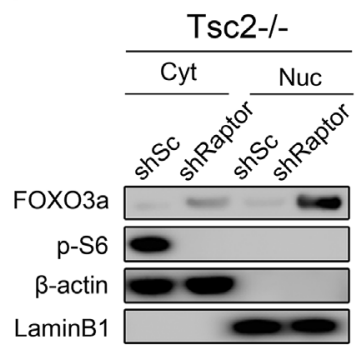

I

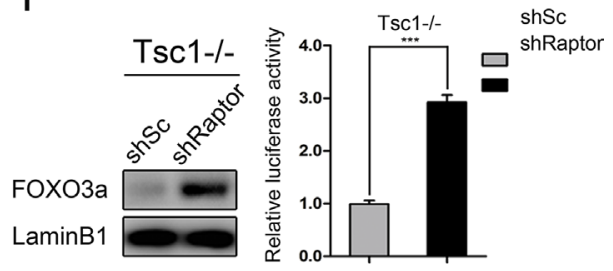

Figure 4: mTORC1 is a negative regulator of FOXO3a. (A) Total cell lysates from Tsc2+/+, Tsc2-/-, rapamycin-treated (20 nM $24 \mathrm{~h})$ Tsc2-/-, Tsc1+/+, Tsc1-/- and rapamycin-treated (20 nM $24 \mathrm{~h})$ Tsc1-/-MEFs were subjected to immunoblotting with the indicated antibodies. (B-D) Tsc2+/+, Tsc2-/-, and rapamycin-treated (20 nM 24 h) Tsc2-/-MEFs. B. The cytoplasm (Cyt) and nuclear (Nuc) proteins were subjected to immunoblotting with the indicated antibodies. C. The expression of FOXO3a was analyzed by an immunofluorescence assay. Scale bar, $50 \mu \mathrm{m}$. D. The cells were co-transfected with pGMFOXO-Luc (200 ng) and the internal control plasmid pRL-TK (20 ng). The relative luciferase activity was measured $24 \mathrm{~h}$ after transfection. (E) Tsc1-/- MEFs were treated with or without rapamycin (20 nM) for $24 \mathrm{~h}$. The nuclear proteins were subjected to immunoblotting with the indicated antibodies (left panel). The relative luciferase activity was measured as D (right panel). (F) Total cell lysates from Tsc2-/- or Tsc1-/- MEFs transduced with shRaptor or shSc lentiviruses were subjected to immunoblotting with the indicated antibodies. G-H. Tsc2-/- MEFs were transduced with shRaptor or shSc lentiviruses. (G) The cytoplasm (Cyt) and nuclear (Nuc) proteins were subjected to immunoblotting with the indicated antibodies. (H) The cells were co-transfected with pGMFOXO-Luc (200 ng) and the internal control plasmid pRL-TK (20 ng). The relative luciferase activity was measured $24 \mathrm{~h}$ after transfection. (I) Tsc1-/- MEFs were infected with shRaptor or shSc lentiviruses. The nuclear proteins were subjected to immunoblotting with the indicated antibodies (left panel). The relative luciferase activity was measured as $\mathrm{H}$ (right panel). $* * P<0.01$; $* * * P<0.001$. 
A
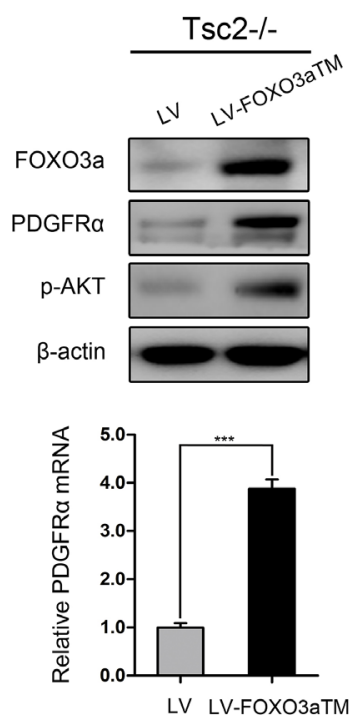

C

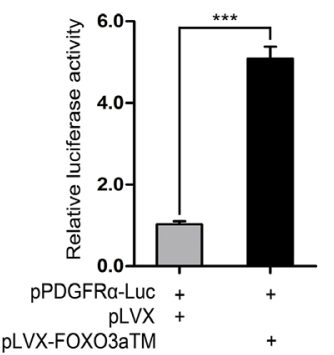

D

D pLVX-FOXO3aTM
E
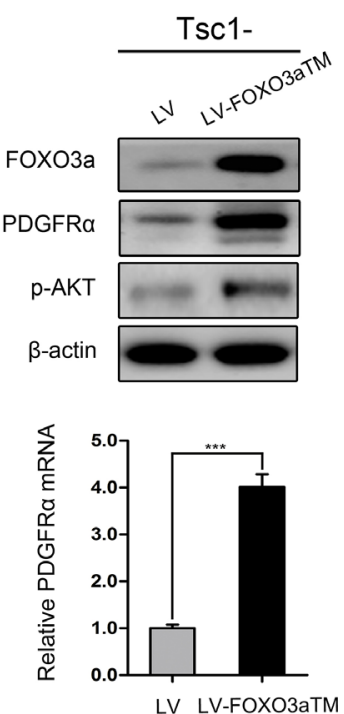

G
B
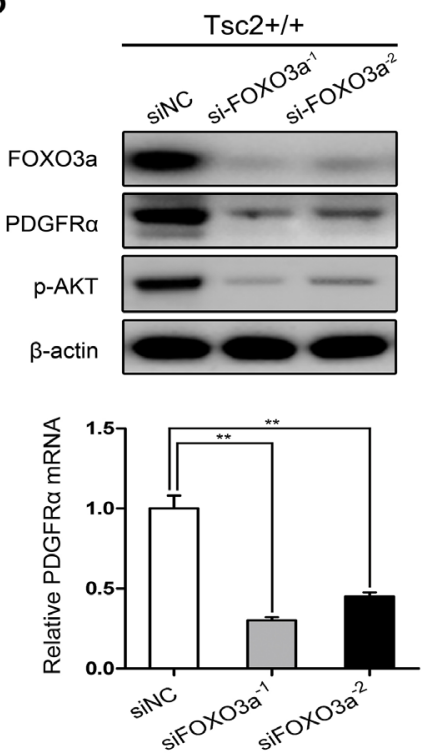
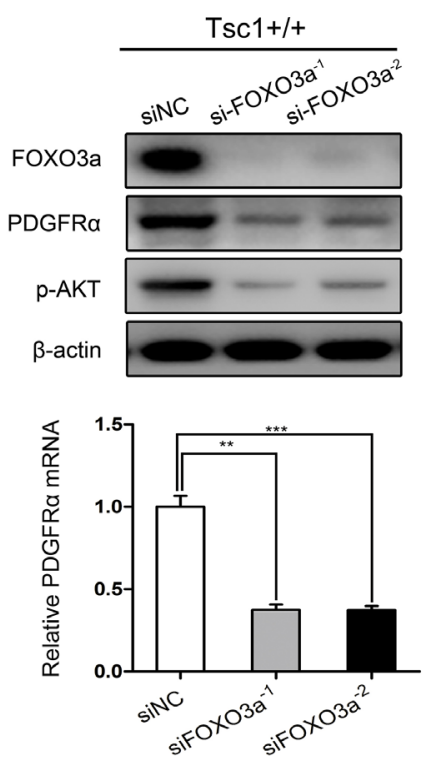

F
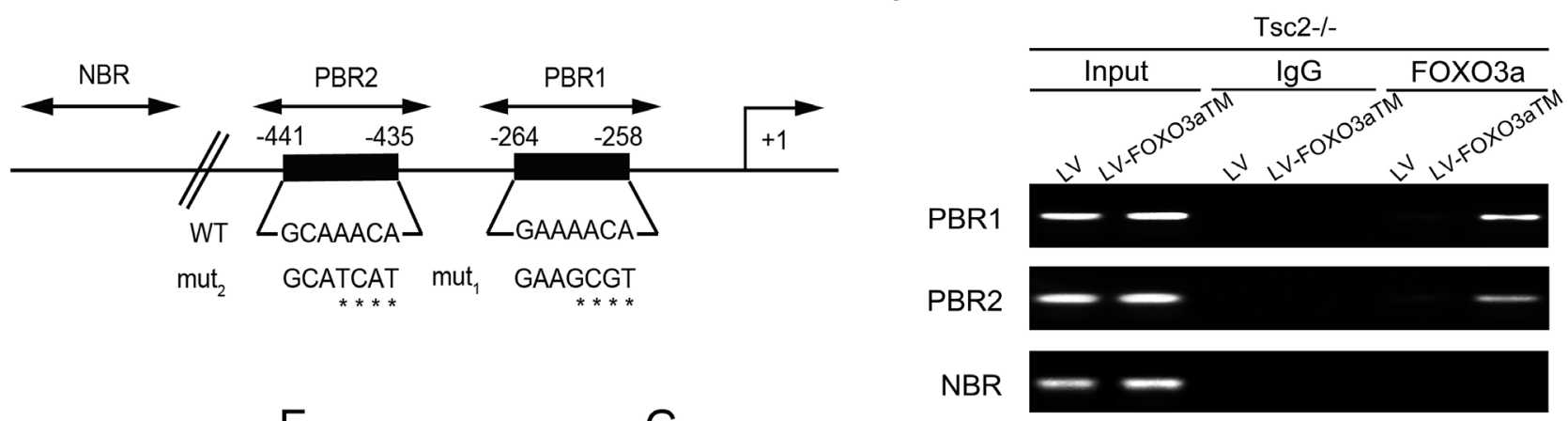

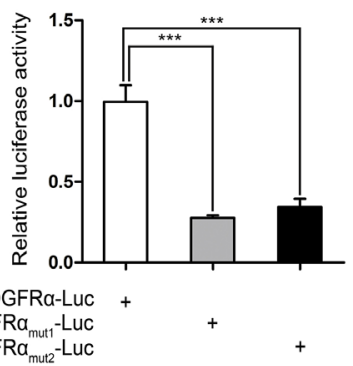

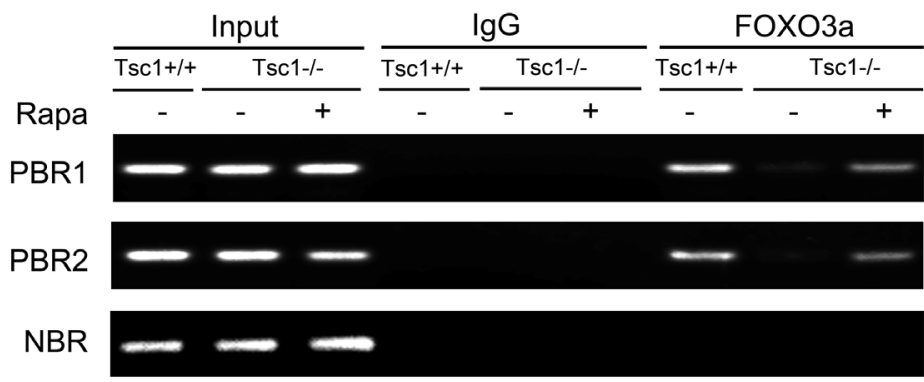

Figure 5: mTORC1 downregulates PDGFRa/AKT pathway through inhibition of FOXO3a. (A) Tsc2-/- or Tsc1-/- MEFs were infected with lentivirus harboring a vector encoding FOXO3aTM (LV-FOXO3aTM) or the empty vector (LV). (B) Tsc2+/+ or Tsc1+/+ MEFs were transfected with two independent siRNAs targeting FOXO3a or the control siRNAs (siNC) for $48 \mathrm{~h}$. A and B. Cell lysates and RNA were subjected to immunoblotting (upper panels) and qRT-PCR (lower panels), respectively. (C) Schematic representation of the putative wild-type (WT) and mutated ( mut $_{1}$ and mut $_{2}$ ) FOXO3a-binding sites in the promoter of mouse PDGFRa gene. (D) HEK293T cells were co-transfected with pPDGFRa-Luc reporter plasmid plus FOXO3aTM expression vector (pLVX-FOXO3aTM) or control vector (pLVX) and the internal control plasmid pRL-TK. (E) Tsc2-/-MEFs were co-transfected with pLVX-FOXO3aTM plus pPDGFRa-Luc, $\mathrm{pPDGFRa}_{\text {mut }}-\mathrm{Luc}$, or pPDGFRa $\mathrm{mut}_{2}-$ Luc reporter plasmid and pRL-TK plasmid. D and E. Relative luciferase activity was examined $24 \mathrm{~h}$ after transfection. (F) Tsc2-/- MEFs were transduced with LV-FOXO3aTM or LV lentiviruses. (G) Tsc1+/+, Tsc1-/-, or rapamycin-treated (20 nM 24 h) Tsc1-/- MEFs. F and G. Cells were subjected to ChIP assay using an anti-FOXO3a antibody. Normal rabbit IgG antibody served as the negative control. PCR was performed to amplify regions surrounding the putative FOXO3a-binding regions (PBR1 and PBR2) and a nonspecific FOXO3a-binding region (NBR). Representative data from two independent experiments were shown. $* * P<0.01$; $* * * P<0.001$. 
regulation of transcription factors, such as STAT3, HIF1 $\alpha$, and etc. [20, 27, 28, 30, 41]. In this study, we demonstrated that the transcription factor FOXO3a, as a downstream target of mTORC1, transcriptionally regulates PDGFR $\alpha$ expression in Tsc1- or Tsc2-deficient cells. This findings not only confirmed a previous study which showed that FOXO3a transcriptionally regulates the expression of PDGFR $\alpha$ in neuroblastoma cells [42], but also revealed that $\mathrm{FOXO} 3 \mathrm{a}$ activity is negatively regulated by mTORC1 (Figure 4), which was consistent with a previous study indicating that inactivation of mTORC1 induces nuclear accumulation of FOXO3a [43]. In addition to suppression of FOXO3a transcriptional activity, mTORC1 inhibited the expression of FOXO3a at both protein and mRNA levels (Figure 4 and Supplementary Figure 2). It has been reported that the expression of FOXO3a could be epigenetically regulated [43]. However, treatment with 5-aza-deoxycytidine (5-aza-dC) or trichostatin A (TSA) had a minimal effect on the expression of FOXO3a in Tsc2-/- MEFs (Supplementary Figure 3), indicating that the epigenetic regulation was not implicated in mTORC1mediated downregulation of FOXO3a in cells lacking TSC1/TSC2 complex. In addition to mTORC1, mTORC2 has also been reported to be involved in the regulation
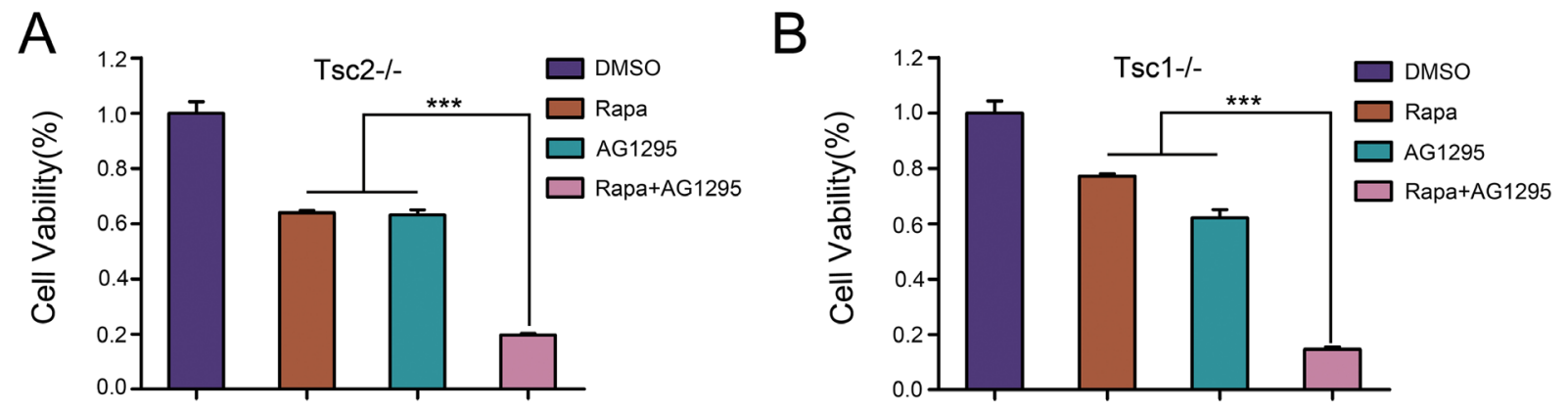

C

D


DMSO

Rapa

AG1295

E

F
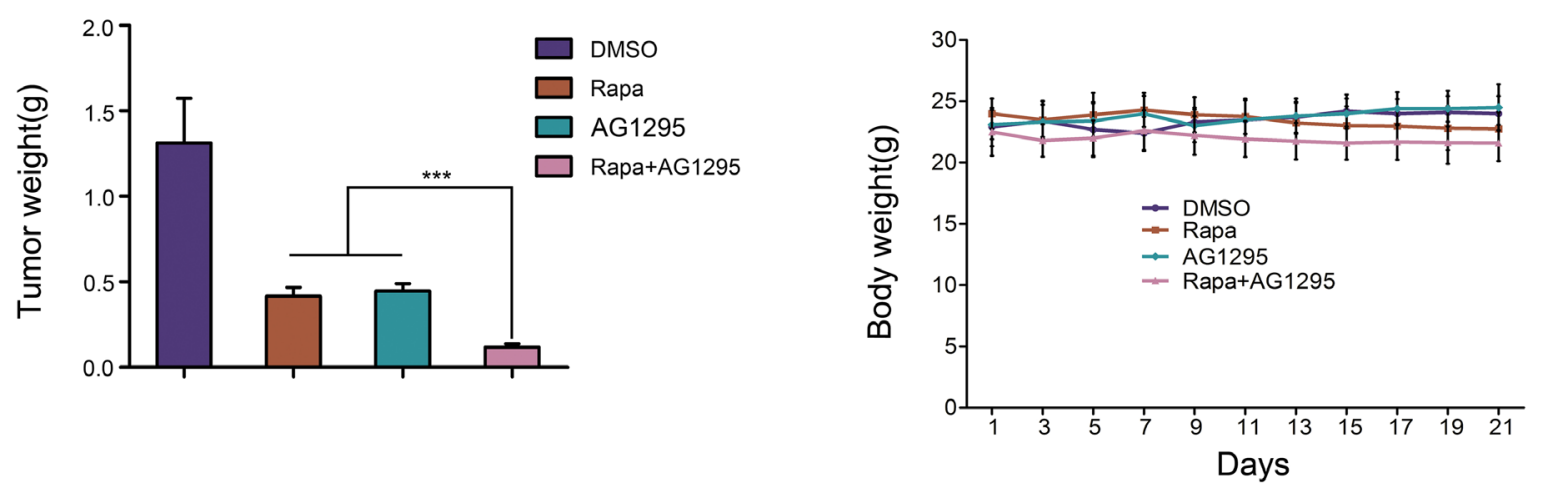

Figure 6: The combination of rapamycin and AG1295 more effectively suppresses the growth of cells lacking TSC1/ TSC2 complex in vitro and in vivo than either agent alone. (A, B) Tsc2-/- (A) or Tsc1-/- MEFs (B) were treated with a combination of $5 \mathrm{nM}$ rapamycin (Rapa) and $20 \mu \mathrm{M}$ AG1295 or either agent alone for $48 \mathrm{~h}$. Cell viability was examined with an MTT assay. (C-F) NTC/T2-null cells were inoculated subcutaneously into the nude mice to evaluate the effects of rapamycin and AG1295 in vivo. (C) Tumor volume growth curves. (D) Dissected tumors. (E) Tumor weight. (F) Body weights of mice. $* * P<0.01 ; * * * P<0.001$. 
of FOXO3a [44-46]. Intriguingly, herein we found that inhibition of mTORC1, but not mTORC2, upregulated the expression level and activity of FOXO3a in Tsc1- or Tsc2deficient MEFs (Figure 4 and Supplementary Figure 4). This discrepancy could be explained by the findings that mTORC2 activity has been inhibited by loss of TSC1/ TSC2 complex [47, 48], and therefore knockdown of Rictor, a specific component of mTORC2, can no longer reduce $\mathrm{mTORC} 2$ activity as well as its downstream targets in Tsc1- or Tsc2-null MEFs.

It is well established that AKT phosphorylation of FOXO3a leads to FOXO3a sequestration in the cytoplasm and inactivates its transcription activities [34]. Interestingly, emerging evidence indicates that FOXO3a can activate AKT. Chen etc. found that constitutively nuclear localization of FOXO3a promotes AKT phosphorylation in breast cancer [49]. Hui and colleagues reported that FOXO3a upregulates AKT activity through induction of PI3KCA expression in drug-resistant leukemic cells [50]. In addition, FOXO3a has been also reported to enhance the expression of Rictor, leading to increased AKT phosphorylation [51, 52]. In line with these previous studies, we found that FOXO3a positively regulates AKT activity in WT cells or Tsc1- or Tsc2-deficient cells (Figure 5). Moreover, PDGFR $\alpha$ was identified as a direct downstream target of FOXO3a, which mediates the activation of AKT (Figure 3 and Figure 5). In addition, we displayed that aberrantly activated mTORC1 inhibits FOXO3a, leading to downregulated expression of PDGFR $\alpha$ and consequently decreased AKT activity. Thus, downregulated FOXO3a/PDGFR $\alpha / \mathrm{AKT}$ pathway may serve as a protective mechanism against TSC-associated tumor development.
Since aberrant activation of mTORC1 is considered to be mainly responsible for TSC-related tumor formation, rapamycin and its analogues have been suggested to be effective drugs for TSC treatment $[9,53-55]$. However, the therapeutic efficacy of such drugs is limited by several reasons, including immunosuppression-associated opportunistic infections [56] and reactivation of AKT [17, 18]. Here we demonstrated that rapamycin treatment upregulated PDGFR $\alpha$ expression (Figure 1), and overexpression of PDGFR $\alpha$ accelerated cell proliferation and tumorigenesis of Tsc1- or Tsc2-null MEFs (Figure 2). It is a widely used strategy for tumor treatment to reduce cytotoxicity and increase treatment efficacy by rational combination of different drugs. So we hypothesized that combined administration of rapamycin and a PDGFR inhibitor would achieve a better therapeutic effect than rapamycin alone in TSC treatment. As shown in Figure 6, combination of rapamycin and AG1295 exhibited a stronger inhibitory effect on the growth of cells lacking TSC1/TSC2 complex in vitro and in vivo. Therefore, co-administration of rapamycin and a PDGFR inhibitor may be an effective and novel strategy for the treatment of TSC.

In conclusion, we have demonstrated that mTORC1 activation due to loss of TSC1/TSC2 complex led to feedback inhibition of AKT signaling through downregulation of FOXO3a/PDGFRa cascade (Figure 7). This study shed new light on the underlying mechanism of benign tumor formation in TSC patients and contributes to a better understand of mechanisms involved in FOXO3amediated activation of AKT. Importantly, the results of our



Figure 7: Schematic illustration of TSC1/TSC2/mTORC1 pathway regulation of PDGFRa/AKT signaling cassette through FOXO3a. Hyperactivation of mTORC1 induced by loss of TSC1/TSC2 complex results in feedback inhibition of AKT signaling through downregulation of FOXO3a/PDGFRa cascade, which contributes to the benign nature of TSC-associated tumors. 
work suggest that combined administration of rapamycin with a PDGFR inhibitor may be a novel strategy for the treatment of TSC-related tumors.

\section{MATERIALS AND METHODS}

\section{Reagents, plasmids, and antibodies}

Rapamycin was obtained from Selleck Chemicals (Houston, TX, USA). Tyrphostin AG1295 was from Santa Cruz Technology (Santa Cruz, CA, USA). 5-azadeoxycytidine (5-aza-dC) and trichostatin A (TSA) were from Sigma-Aldrich (St Louis, MO, USA). Murine PDGFAA was acquired from Pepro Tech (Rocky Hill, NJ, USA). Fetal bovine serum (FBS), Dulbecco's Modified Eagle's Medium (DMEM), Lipofectamine 3000, and NuPAGE 4-12\% Bis-Tris Gels were purchased from Life Technologies (Carlsbad, CA, USA). pLVX-IRES-Puro vector was from Clontech (Mountain View, CA, USA). The FOXO luciferase reporter plasmid pGMFOXO-Luc was purchased from Genomeditech (Shanghai, China). A constitutively active FOXO3a (FOXO3aTM), in which all three AKT phosphorylation sites were mutated to alanine, was obtained from Addgene (Cambridge, MA, USA). Antibodies against TSC1, Raptor, Rictor, phospho-S6 (Ser235/236), PDGFRa, FOXO3a, AKT1 and phospho-AKT (Ser473) were from Cell Signaling Technology (Danvers, MA, USA). LaminB1, phospho-FOXO3a (Ser253) and Ki-67 antibodies were from Abcam (Cambridge, MA, USA). TSC2, $\beta$-actin, and all HRP-labeled secondary antibodies were from Santa Cruz Technology.

\section{Cell culture}

Tsc2+/+, Tsc2-/-, Tsc1+/+, Tsc1-/- MEFs, and NTC/T2-null cells have been described previously $[17,27,28]$. HEK293T cells were obtained from the ATCC (Manassas, VA, USA). All cells were maintained and propagated in DMEM with 10\% FBS and 1\% penicillin/ streptomycin in $5 \% \mathrm{CO}_{2}$ at $37^{\circ} \mathrm{C}$.

\section{Cell fractionation and immunoblotting}

Cytoplasmic and nuclear protein fractions were extracted with a NE-PER Reagent Kit (Pierce, Rockford, IL, USA) according to the manufacturer's instructions. Cell or tumor tissue lysates were separated by NuPAGE 4-12\% Bis-Tris Gels (Life Technologies), and then transferred to PVDF membrane (Millipore, Billerica, MA, USA), followed by incubation with appropriate primary antibodies and horseradish peroxidase (HRP)-conjugated secondary antibodies. Immunoreactive bands were visualized with Pierce ${ }^{\mathrm{TM}}$ ECL Western Blotting Substrate (Thermo Scientific, Waltham, MA, USA) according to the manufacturer's protocol.

\section{Quantitative real time-PCR (qRT-PCR)}

Total RNA was isolated from cells using TRIzol reagent (Life Technologies) according to the manufacturer's instructions. $1 \mu \mathrm{g}$ of RNA was converted into cDNA using the RevertAid ${ }^{\mathrm{TM}}$ First Stand cDNA Synthesis Kit (Fermentas, Waltham, MA, USA). After 10-fold dilution, $4 \mu \mathrm{l}$ of cDNA was subjected to PCR amplification using SYBR Premix Ex Taq ${ }^{\text {TM }}$ II (TaKaRa, Shiga, Japan) according to the manufacturer's protocol in a StepOnePlus ${ }^{\mathrm{TM}}$ Real-Time PCR System (ABI, Foster City, CA, USA). $\beta$-actin served as the internal control. The primer sequences were as follows: PDGFRa forward, 5'-ACACGTTTGAGCTGTCAACC-3', and reverse, 5'-CCCGACCACACAAGAACAGG-3'; FOXO3a forward, 5'-CTGGGGGAACCTGTCCTATG3', and reverse, 5'-TCATTCTGAACGCGCATGAAG-3'; $\beta$-actin forward, 5'-AGAAAATCTGGCACCACACC-3', and reverse, 5'-AGAGGCGTACAGGGATAGCA-3'.

\section{RNA interference}

Cells were seeded into 12-well plates and transfected with siRNAs using siRNA-Mate (GenePharma, Shanghai, China) according to the manufacturer's protocol. All siRNA oligonucleotides were synthesized by GenePharma. The siRNA target sequences were as follows: Raptor, 5'-GGACAACGGTCACAAGTAC-3'; FOXO3a ${ }^{-1}, 5^{\prime}$-CAT GCGCGTTCAGAATGAA-3'; FOXO3a ${ }^{-2}, 5^{\prime}$-GAACG TTGTTGGTTTGAAT-3'; Negative Control (NC), 5'-TTC TCCGAACGTGTCACGT-3'.

\section{Preparation of recombinant plasmids and viruses}

GV248 lentiviral shRNA expression vectors targeting mouse PDGFRa, mouse Raptor, mouse Rictor, and the control scrambled shRNA (shSc) were obtained from Genechem (Shanghai, China). The target sequence for PDGFRa was 5'-CCTGGAGAAGTGAGAAACAAA-3'. The target sequence for Rictor was 5'-GCCCTA CAGCCTTCATTTA- $3^{\prime}$. The target sequence for Raptor is consistent with the sequences used in RNA Interference as above. GV367 lentiviral plasmid expressing mouse PDGFRa and the empty plasmid were obtained from Genechem (Shanghai, China). The FOXO3aTM expression vector was constructed by subcloning FOXO3aTM cDNA (Addgene) into the pLVX-IRES-Puro vector. All recombinant plasmids were verified by DNA sequencing. The recombinant plasmids and empty control vector were named as LV-PDGFRa, LV-FOXO3aTM, and LV, respectively. Lentiviruses were generated by co-transfecting a recombinant vector or the corresponding control vector with the packaging vectors (pVSVG, pREV, and pMDL) into HEK293T cells. After $48 \mathrm{~h}$ of transfection, culture supernatants were collected and filtered with a $0.45 \mu \mathrm{m}$ filter, and then used to infect target cells as described previously [27]. 


\section{Cell proliferation and viability assay}

Cell proliferation was measured by an MTT assay according to the manufacturer's instructions and has been described previously [29]. For the cell viability assays, Tsc1- or Tsc2-null MEFs were seeded in 96-well plates at a density of 3, 000 cells/well and treated with DMSO, rapamycin, AG1295, or combination of rapamycin and AG1295 for $48 \mathrm{~h}$. Followed by added $10 \mu \mathrm{l}$ of Cell Counting Kit-8 reagent (Beyotime, China) to per well and incubated the plates for $1 \mathrm{~h}$, the optical density (OD value) of each well was measured at $450 \mathrm{~nm}$.

\section{Colony formation assay}

Cells were seeded into $100 \mathrm{~mm}$ cell culture dish at a density of 200 cells per dish. After incubation for approximately 2 weeks in DMEM containing 10\% FBS, the cells were fixed and dyed with $0.1 \%$ crystal violet $(1 \mathrm{mg} / \mathrm{ml})$. The number of colonies containing over 50 cells was counted.

\section{Immunofluorescence assay}

FOXO3a was analyzed by immunofluorescence assay. Briefly, cells were seeded on coverslips overnight and then fixed with $4 \%$ formaldehyde for $5 \mathrm{~min}$ at room temperature, and followed by the treatment of $1 \%$ Triton X-100 (Sigma-Aldrich) for permeabilization. After blocking with $2 \%$ bovine serum albumin for $1 \mathrm{~h}$, the cells were incubated with anti-FOXO3a antibody (Cell Signaling Technology) for overnight and then incubated with FITC-conjugated secondary antibody (Cell Signaling Technology) for $1 \mathrm{~h}$. DAPI (Sigma-Aldrich) was used to visualize cell nucleus and the fluorescence staining was examined under a FV1000 confocal microscope (Olympus, Tokyo, Japan).

\section{Induction of subcutaneous tumors and combination treatment of rapamycin and AG1295}

Immunodeficient $\mathrm{BALB} / \mathrm{c}$ nude mice (5 weeks old) were purchased from Vital River Laboratory Animal Technology (Beijing, China). $3 \times 10^{6}$ cells as the indicated in $0.2 \mathrm{ml}$ DMEM were inoculated subcutaneously into the right anterior armpit of mice and the tumor growth was monitored. Five mice were used in each cohort. The mice were euthanized and imaged on day 50 after inoculation. Subcutaneous tumors were established as described previously $[18,30]$.

To evaluate the therapeutic efficacy of rapamycin and AG1295 in vivo, NTC/T2-null cells $\left(3 \times 10^{6}\right.$ cells $)$ were injected subcutaneously near the axillary fossa of nude mice. The mice were randomly divided into four groups (five mice per group) when the tumor volumes reached $50-70 \mathrm{~mm}^{3}$. And then the mice in four different groups were treated with vehicle solution (75\% DMSO and $25 \% \mathrm{PBS})$, rapamycin (2 mg/kg), AG1295 (5 mg/kg), or combination of rapamycin $(2 \mathrm{mg} / \mathrm{kg})$ and AG1295 $(5 \mathrm{mg} / \mathrm{kg})$, respectively (i.p., every two days, a total of ten injections). Tumor dimensions were measured with a digital caliper every two days, and the tumor volume was calculated using the formula $\mathrm{V}=1 / 2$ ( width $^{2} \times$ length). Body weights were also monitored. On day 34 after inoculation of tumor cells, all experimental mice were terminated with ether anesthesia and the total weight of tumors in mice were measured. All animals were maintained and used in accordance with the guidelines of the Animal Center of Anhui Medical University, and all animal experimental procedures were approved by the Experimental Animal Ethical Committee of Anhui Medical University.

\section{Immunohistochemistry (IHC)}

Immunohistochemical analysis was performed as described previously [31]. In brief, tumor tissues were fixed in 4\% paraformaldehyde and embedded in paraffin. Sections $(4 \mu \mathrm{m})$ were prepared for the indicated primary antibodies or hematoxylin and eosin (H\&E) staining according to standard protocols.

\section{Promoter-reporter constructs and luciferase reporter assay}

A 650-bp fragment of the mouse PDGFRa promoter $(-582 /+68)$ was obtained by PCR using mouse genomic DNA extracted from Tsc2+/+ MEFs. Primer sequences used were as follows: forward, 5'-TTGAAATGCGTGCAAACGCTGAGCATAG-3'; reverse, 5'-CTGCGACCTGAGAGGAGACTGGGCA-3'. The amplified DNA fragments were cloned into the pGL3Basic (Promega, Madison, WI, USA) plasmid through the Kpn I and Xho III sites. The potential FOXO3abinding sites on the promoter of mouse PDGFRa gene were mutated using the Q5 Hot Start High-Fidelity DNA Polymerase (NEB, Ipswich, MA, USA). The primer sequences were as follows: PDGFRa $a_{\text {mut }}$, forward, 5'-AGGGGCAGGGCATCATAAGGGGCAAGGT-3', reverse, 5'-GGGCACCCCTGCTCTACTTCATGCCT CTA-3'; PDGFRa $a_{\text {mut } 2}$, forward, 5'-TCGCAGTTGAAGC GTATGCAAACGCTGA-3', reverse, 5'-CCTGAGAGG AGACTGGGCAGGGTGGA-3'. For Luciferase reporter assays, cells were cultured in triplicate to $80 \%$ confluence in 24-well plates and co-transfected with the promoter constructs (200 ng) and the internal control plasmid pRLTK (20 ng). Luciferase activity was detected with the Dual-Luciferase Reporter Assay System (Promega).

\section{Chromatin immunoprecipitation (ChIP)}

ChIP assay with an anti-FOXO3a antibody (Abcam) to detect protein-DNA interactions were 
performed using a SimpleChIP ${ }^{\circledR}$ Plus Enzymatic Chromatin IP kit (Cell Signaling Technology) according to manufacturer's protocol. The purified DNA was analyzed by standard PCR with Hot Start Taq DNA Polymerase (NEB). The primer sequences were as follows: the putative FOXO3a-binding region 1 (PBR1), forward, 5'-TGTGGCTCGCCTGTAACCCTAAAC-3', reverse, 5'-ATACCAGGCGACACGGATCTACCC-3'; the putative FOXO3a-binding region 2 (PBR2), forward, 5'-GGGGATAAATGTAAGCCACGGACT-3', reverse, 5'-CTGGACCAAGCTCTGTGATTCGG-3'; a nonspecific FOXO3a-binding region (NBR), forward, 5'-GGAAGTGTTGGCCTGTTTGTCTGC-3', reverse, 5'-TCTGGGCAGCTCTGTCCGACG-3'.

\section{Statistics}

All quantitative data were represented as mean \pm SEM of at least three independent experiments. The difference between two groups was evaluated with 2 -tailed Student's $t$-test. One-way ANOVA and Tukey post hoc test were used to evaluate differences of multiple comparisons. All statistical analyses were conducted using GraphPad Prism software. Differences were considered significant when $p<0.05$.

\section{Abbreviations}

TSC: tuberous sclerosis complex; mTOR: mammalian target of rapamycin; mTORC1: mammalian target of rapamycin complex 1; mTORC2: mammalian target of rapamycin complex 2; PDGFR $\alpha$ : platelet-derived growth factor receptor $\alpha$; FOXO3a: forkhead box O3a; MEFs: mouse embryonic fibroblasts.

\section{Authors' contributions}

X.Z., Z.H. and H.Z. designed the study; L.W., Z.N., Y.L., S.J., F.J., K.J. and C.R. performed the experiments; L.W., Z.N., Y.L., J.M., Z.H. and X.Z. performed data analysis and interpretation; X.Z. and Z.H. wrote the paper.

\section{ACKNOWLEDGMENTS}

We thank Haisheng Zhou (Anhui Medical University) for technical assistance in this study.

\section{CONFLICTS OF INTEREST}

The authors declare no conflicts of interest.

\section{FUNDING}

This work was supported by the National Natural Science Foundation of China $(81372475,81101524$, and 81100876), the Key Program in the Youth Elite Support
Plan in Universities of Anhui Province (for X. Zha), the Outstanding Young Scholars Plan of Anhui Medical University (for X. Zha), Anhui Provincial Natural Science Foundation (1408085MH157), and Excellent Young Scientist Foundation of Beijing University of Chinese Medicine (2015-JYB-XYQ-004).

\section{REFERENCES}

1. Laplante M, Sabatini DM. mTOR signaling in growth control and disease. Cell. 2012; 149:274-293.

2. Betz C, Hall MN. Where is $\mathrm{mTOR}$ and what is it doing there? J Cell Biol. 2013; 203:563-574.

3. Guertin DA, Sabatini DM. Defining the role of mTOR in cancer. Cancer Cell. 2007; 12:9-22.

4. Kim YC, Guan KL. mTOR: a pharmacologic target for autophagy regulation. J Clin Invest. 2015; 125:25-32.

5. Laplante M, Sabatini DM. mTOR signaling at a glance. J Cell Sci. 2009; 122:3589-3594.

6. Toschi A, Lee E, Xu L, Garcia A, Gadir N, Foster DA. Regulation of mTORC1 and mTORC 2 complex assembly by phosphatidic acid: competition with rapamycin. Mol Cell Biol. 2009; 29:1411-1420.

7. Alessi DR, Pearce LR, Garcia-Martinez JM. New insights into mTOR signaling: mTORC2 and beyond. Sci Signal. 2009; 2:pe27.

8. Hay N, Sonenberg N. Upstream and downstream of mTOR. Genes Dev. 2004; 18:1926-1945.

9. Crino PB, Nathanson KL, Henske EP. The tuberous sclerosis complex. N Engl J Med. 2006; 355:1345-1356.

10. Kwiatkowski DJ, Manning BD. Molecular basis of giant cells in tuberous sclerosis complex. N Engl J Med. 2014; 371:778-780.

11. Goldberg AA, Joung KB, Mansuri A, Kang Y, Echavarria R, Nikolajev L, Sun Y, Yu JJ, Laporte SA, Schwertani A, Kristof AS. Oncogenic effects of urotensin-II in cells lacking tuberous sclerosis complex-2. Oncotarget. 2016; 7:61152-61165. https://doi.org/10.18632/oncotarget.10748.

12. Huang J, Manning BD. The TSC1-TSC2 complex: a molecular switchboard controlling cell growth. Biochem J. 2008; 412:179-190.

13. Liang S, Salas T, Gencaslan E, Li B, Habib SL. Tuberin-deficiency downregulates $\mathrm{N}$-cadherin and upregulates vimentin in kidney tumor of TSC patients. Oncotarget. 2014; 5:6936-6946. https://doi.org/10.18632/ oncotarget.2206.

14. Inoki $\mathrm{K}, \mathrm{Li} \mathrm{Y}, \mathrm{Zhu} \mathrm{T}, \mathrm{Wu} \mathrm{J}$, Guan KL. TSC2 is phosphorylated and inhibited by Akt and suppresses mTOR signalling. Nat Cell Biol. 2002; 4:648-657.

15. Kwiatkowski DJ, Zhang H, Bandura JL, Heiberger KM, Glogauer M, el-Hashemite N, Onda H. A mouse model of TSC1 reveals sex-dependent lethality from liver hemangiomas, and up-regulation of p70S6 kinase activity in Tsc1 null cells. Hum Mol Genet. 2002; 11:525-534. 
16. El-Hashemite N, Zhang H, Henske EP, Kwiatkowski DJ. Mutation in TSC2 and activation of mammalian target of rapamycin signalling pathway in renal angiomyolipoma. Lancet. 2003; 361:1348-1349.

17. Zhang H, Cicchetti G, Onda H, Koon HB, Asrican K, Bajraszewski N, Vazquez F, Carpenter CL, Kwiatkowski DJ. Loss of Tsc1/Tsc2 activates mTOR and disrupts PI3K-Akt signaling through downregulation of PDGFR. J Clin Invest. 2003; 112:1223-1233.

18. Zhang H, Bajraszewski N, Wu E, Wang H, Moseman AP, Dabora SL, Griffin JD, Kwiatkowski DJ. PDGFRs are critical for PI3K/Akt activation and negatively regulated by mTOR. J Clin Invest. 2007; 117:730-738.

19. Manning BD, Logsdon MN, Lipovsky AI, Abbott D, Kwiatkowski DJ, Cantley LC. Feedback inhibition of Akt signaling limits the growth of tumors lacking Tsc2. Genes Dev. 2005; 19:1773-1778.

20. Zha X, Hu Z, He S, Wang F, Shen H, Zhang H. TSC1/TSC2 inactivation inhibits AKT through mTORC1-dependent up-regulation of STAT3-PTEN cascade. Cancer Lett. 2011; 313:211-217.

21. Heldin $\mathrm{CH}$, Westermark B. Mechanism of action and in vivo role of platelet-derived growth factor. Physiol Rev. 1999; 79:1283-1316.

22. Andrae J, Gallini R, Betsholtz C. Role of platelet-derived growth factors in physiology and medicine. Genes Dev. 2008; 22:1276-1312.

23. Heldin $\mathrm{CH}$. Targeting the PDGF signaling pathway in tumor treatment. Cell Commun Signal. 2013; 11:97.

24. Wang F, Moseman A, Doucette M, Bhat K, Li X, Tian Z, Zhang H, Law SH, Kohane I, Wu E. PDGFR alpha and PDGFR beta differentially regulate cell proliferation and migration/invasion in medulloblastoma cells. Cancer Res. 2010; 70:288.

25. Zhang J, Cao R, Zhang Y, Jia T, Cao Y, Wahlberg E. Differential roles of PDGFR-alpha and PDGFR-beta in angiogenesis and vessel stability. FASEB J. 2009; 23:153-163.

26. Ehnman M, Missiaglia E, Folestad E, Selfe J, Strell C, Thway K, Brodin B, Pietras K, Shipley J, Ostman A, Eriksson U. Distinct effects of ligand-induced PDGFRalpha and PDGFRbeta signaling in the human rhabdomyosarcoma tumor cell and stroma cell compartments. Cancer Res. 2013; 73:2139-2149.

27. Ma J, Meng Y, Kwiatkowski DJ, Chen X, Peng H, Sun Q, Zha X, Wang F, Wang Y, Jing Y, Zhang S, Chen R, Wang L, et al. Mammalian target of rapamycin regulates murine and human cell differentiation through STAT3/p63/Jagged/ Notch cascade. J Clin Invest. 2010; 120:103-114.

28. Zha X, Wang F, Wang Y, He S, Jing Y, Wu X, Zhang H. Lactate dehydrogenase $\mathrm{B}$ is critical for hyperactive mTORmediated tumorigenesis. Cancer Res. 2011; 71:13-18.

29. Li H, Jin F, Jiang K, Ji S, Wang L, Ni Z, Chen X, Hu Z, Zhang H, Liu Y, Qin Y, Zha X. mTORC1-mediated downregulation of COX2 restrains tumor growth caused by TSC2 deficiency. Oncotarget. 2016; 7:28435-28447. https:// doi.org/10.18632/oncotarget.8633.
30. Sun Q, Chen X, Ma J, Peng H, Wang F, Zha X, Wang Y, Jing Y, Yang H, Chen R, Chang L, Zhang Y, Goto J, et al. Mammalian target of rapamycin up-regulation of pyruvate kinase isoenzyme type M2 is critical for aerobic glycolysis and tumor growth. Proc Natl Acad Sci USA. 2011; 108:4129-4134.

31. Peng H, Liu J, Sun Q, Chen R, Wang Y, Duan J, Li C, Li B, Jing Y, Chen X, Mao Q, Xu KF, Walker CL, et al. mTORC1 enhancement of STIM1-mediated store-operated $\mathrm{Ca} 2+$ entry constrains tuberous sclerosis complex-related tumor development. Oncogene. 2013; 32:4702-4711.

32. Greer EL, Brunet A. FOXO transcription factors in ageing and cancer. Acta Physiol (Oxf). 2008; 192:19-28.

33. Park SH, Chung YM, Ma J, Yang Q, Berek JS, Hu MC. Pharmacological activation of FOXO3 suppresses triplenegative breast cancer in vitro and in vivo. Oncotarget. 2016; 7:42110-42125. https://doi.org/10.18632/oncotarget.9881.

34. Fu Z, Tindall DJ. FOXOs, cancer and regulation of apoptosis. Oncogene. 2008; 27:2312-2319.

35. Manning BD, Cantley LC. AKT/PKB signaling: navigating downstream. Cell. 2007; 129:1261-1274.

36. Henske EP, Jozwiak S, Kingswood JC, Sampson JR, Thiele EA. Tuberous sclerosis complex. Nat Rev Dis Primers. 2016; 2:16035.

37. Tyburczy ME, Jozwiak S, Malinowska IA, Chekaluk Y, Pugh TJ, Wu CL, Nussbaum RL, Seepo S, Dzik T, Kotulska K, Kwiatkowski DJ. A shower of second hit events as the cause of multifocal renal cell carcinoma in tuberous sclerosis complex. Hum Mol Genet. 2015; 24:1836-1842.

38. Kristof AS, Li PZ, Major P, Landry JS. Lymphangioleiomyomatosis and Tuberous Sclerosis Complex in Quebec: Prevalence and Health-care Utilization. Chest. 2015; 148:444-449.

39. Yu Y, Yoon SO, Poulogiannis G, Yang Q, Ma XM, Villen J, Kubica N, Hoffman GR, Cantley LC, Gygi SP, Blenis J. Phosphoproteomic analysis identifies Grb10 as an mTORC1 substrate that negatively regulates insulin signaling. Science. 2011; 332:1322-1326.

40. Jin F, Jiang K, Ji S, Wang L, Ni Z, Huang F, Li C, Chen R, Zhang H, Hu Z, Zha X. Deficient TSC1/TSC2-complex suppression of SOX9-osteopontin-AKT signalling cascade constrains tumour growth in tuberous sclerosis complex. Hum Mol Genet. 2017; 26:407-419.

41. Hu Z, Wang Y, Huang F, Chen R, Li C, Wang F, Goto J, Kwiatkowski DJ, Wdzieczak-Bakala J, Tu P, Liu J, Zha X, Zhang H. Brain-expressed X-linked 2 Is Pivotal for Hyperactive Mechanistic Target of Rapamycin (mTOR)-mediated Tumorigenesis. J Biol Chem. 2015; 290:25756-25765.

42. Mei Y, Wang Z, Zhang L, Zhang Y, Li X, Liu H, Ye J, You H. Regulation of neuroblastoma differentiation by forkhead transcription factors FOXO1/3/4 through the receptor tyrosine kinase PDGFRA. Proc Natl Acad Sci USA. 2012; 109:4898-4903.

43. Mori S, Nada S, Kimura H, Tajima S, Takahashi Y, Kitamura A, Oneyama C, Okada M. The mTOR pathway 
controls cell proliferation by regulating the FoxO3a transcription factor via SGK1 kinase. PLoS One. 2014; 9:e88891.

44. Calnan DR, Brunet A. The FoxO code. Oncogene. 2008; 27:2276-2288.

45. Masui K, Tanaka K, Akhavan D, Babic I, Gini B, Matsutani T, Iwanami A, Liu F, Villa GR, Gu Y, Campos C, Zhu S, Yang H, et al. mTOR complex 2 controls glycolytic metabolism in glioblastoma through FoxO acetylation and upregulation of c-Myc. Cell Metab. 2013; 18:726-739.

46. Guertin DA, Stevens DM, Thoreen CC, Burds AA, Kalaany NY, Moffat J, Brown M, Fitzgerald KJ, Sabatini DM. Ablation in mice of the mTORC components raptor, rictor, or $\mathrm{mLST} 8$ reveals that $\mathrm{mTORC} 2$ is required for signaling to Akt-FOXO and PKCalpha, but not S6K1. Dev Cell. 2006; 11:859-871.

47. Yang Q, Inoki K, Kim E, Guan KL. TSC1/TSC2 and Rheb have different effects on TORC1 and TORC2 activity. Proc Natl Acad Sci USA. 2006; 103:6811-6816.

48. Huang J, Dibble CC, Matsuzaki M, Manning BD. The TSC1-TSC2 complex is required for proper activation of mTOR complex 2. Mol Cell Biol. 2008; 28:4104-4115.

49. Chen J, Gomes AR, Monteiro LJ, Wong SY, Wu LH, $\mathrm{Ng}$ TT, Karadedou CT, Millour J, Ip YC, Cheung YN, Sunters A, Chan KY, Lam EW, et al. Constitutively nuclear FOXO3a localization predicts poor survival and promotes Akt phosphorylation in breast cancer. PLoS One. 2010; 5:e12293.

50. Hui RC, Gomes AR, Constantinidou D, Costa JR, Karadedou CT, Fernandez de Mattos S, Wymann MP,
Brosens JJ, Schulze A, Lam EW. The forkhead transcription factor FOXO3a increases phosphoinositide-3 kinase/Akt activity in drug-resistant leukemic cells through induction of PIK3CA expression. Mol Cell Biol. 2008; 28:5886-5898.

51. Chen CC, Jeon SM, Bhaskar PT, Nogueira V, Sundararajan D, Tonic I, Park Y, Hay N. FoxOs inhibit mTORC1 and activate Akt by inducing the expression of Sestrin3 and Rictor. Dev Cell. 2010; 18:592-604.

52. Lin A, Piao HL, Zhuang L, Sarbassov dos D, Ma L, Gan B. FoxO transcription factors promote AKT Ser473 phosphorylation and renal tumor growth in response to pharmacologic inhibition of the PI3K-AKT pathway. Cancer Res. 2014; 74:1682-1693.

53. Curatolo P, Moavero R. mTOR Inhibitors in Tuberous Sclerosis Complex. Curr Neuropharmacol. 2012; 10:404- 415.

54. Habib SL, Al-Obaidi NY, Nowacki M, Pietkun K, Zegarska B, Kloskowski T, Zegarski W, Drewa T, Medina EA, Zhao Z, Liang S. Is mTOR Inhibitor Good Enough for Treatment All Tumors in TSC Patients? J Cancer. 2016; 7:1621-1631.

55. Zhang ZQ, Shen C, Long Q, Yang ZK, Dai RP, Wang J, Zhang W, Pan Q, Zhu Z, Xu KF. Sirolimus for Retinal Astrocytic Hamartoma Associated with Tuberous Sclerosis Complex. Ophthalmology. 2015; 122:1947-1949.

56. Bissler JJ, McCormack FX, Young LR, Elwing JM, Chuck G, Leonard JM, Schmithorst VJ, Laor T, Brody AS, Bean J, Salisbury S, Franz DN. Sirolimus for angiomyolipoma in tuberous sclerosis complex or lymphangioleiomyomatosis. N Engl J Med. 2008; 358:140-151. 